\title{
CIENCIA\&SALUD
}

\section{TROMBOEMBOLISMO PULMONAR MASIVO SÍNDROME}

ANTIFOSFOLIPIDICO PRIMARIO EN HOMBRE JOVEN

MASSIVE PULMONARY THROMBOEMBOLISM PRIMARY ANTI-

\section{PHOSPHOLIPID SYNDROME}

Alice Umaña Venegas ${ }^{1}$ Christian García Quirós²Anita Valverde Naranjo4Mariam Ramírez Solano ${ }^{4}$

1, 3 y 4 Médico general, Caja costarricense del seguro social San José, Costa Rica. 2, Especialista de Medicina Interna, Caja costarricense de seguro social, San José, Costa Rica.

Contactos: aliceuvu@gmail.com,chrisgarqui@yahoo.es, anitiux19@gmail.com maraso09@hotmail.com

\section{RESUMEN}

Masculino de 37 años, desconocido enfermo, que presenta tromboemobolismo pulmonar masivo, secundario a síndrome antifosfolípido primario.

Palabras Clave:

Síndrome antifosfolipídico, tromboembolismo pulmonar masivo, anticoagulación.

\section{Cómo citar:}

Umaña Venegas, A., García Quirós, C., Valverde Naranjo, A., \& Ramírez Solano, $M$. (2021). TROMBOEMBOLISMO PULMONAR MASIVO. Revista Ciencia Y Salud, 5(2), Pág. 87-95.

Recibido: 7/feb/2021

Aceptado: 30/mar/2021

Publicado: 16/abr/2021

\section{ABSTRACT}

A healthy, 37-year-old male presenting with primary antiphospholipid syndrome, in the context of massive pulmonary thromboembolism, as a descending factor.

\section{Keywords:}

Antiphospholipid syndrome, massive pulmonary thromboembolism, anticoagulation. 


\section{CASO CLÍNICO:}

Masculino de 37 años, vecino de Cartago, desconocido enfermo. El paciente consultó por un cuadro subagudo de aproximadamente un mes de evolución de disnea progresiva de moderados y grandes esfuerzos físicos, acompañado por hemoptisis, sensación febril no cuantificada, con reagudizacion de disnea, incluso en reposo asociado con dolor torácico opresivo retroesternal, irradiado a epigastrio. Por esto, el paciente consulta en múltiples oportunidades y se maneja como IVRS con tratamiento con claritromicina.

Es valorado en el SEM del Hospital Maximiliano Peralta, donde se observa taquipneico, asociado con disnea y saturando $97 \%$ con reservorio. Además, se le realizan pruebas de laboratorio, las cuales documentan los siguientes resultados: hemoglobina $18,3 \mathrm{~g} / \mathrm{dl}$, hematocrito $57 \%$, plaquetas 92000 por ml, leucocitos 11130 , segmentados $67 \%$, nitrógeno ureico $31 \mathrm{mg} / \mathrm{dl}$, creatinina $1,21 \mathrm{mg} / \mathrm{dl}$, sodio $135 \mathrm{meq}$, potasio $4,31 \mathrm{meq}$, presepsina 811, bnp (péptido natriuretico) 8453, tiempo de protrombina 13.8, porcentaje de actividad tp $72 \%$, índice internacional normal (INR)1.23, tiempo parcial de tromboplastina 48.4, bilirrubina total 1.81, bilirrubina indirecta 1.13, bilirrubina directa 0.68 .

El electrocardiograma registra una taquicardia sinusal, sin alteraciones en el PR, QRS con morfología de BRDHH con ST y onda T sin alteraciones. Los gases arteriales documentan acidosis metabólica con hipoxemia severa e hiperlactatemia.

Los hallazgos clínicos conducen a realizar el angiotac documentando TEP Masivo, con atelectasia basal izquierda. Se tromboliza al paciente y se traslada a la $\mathrm{UCl}$, iniciando estudios por síndrome de hipercoagulabilidad. El paciente presenta un índice de severidad de embolismo pulmonar (PESI) de 127 puntos clase VI; por tanto, posterior a trombolisis, se inicia tratamiento anticoagulante con enoxaparina $(5,6,7)$. Él cursa con cánula de alto flujo, con mejoría de los requerimientos de oxígeno suplementario y por ende, su disnea. Se le realiza POCUS (point of care ultrasound), el cual arroja los siguientes hallazgos: patrón A pulmonar derecho, patrón B en base pulmonar izquierda, sin derrame pleural, con contractibilidad global que impresiona estar conservada, con dilatación de cámaras derechas (imagen 1 y 2), D shape (imagen 2), sin derrame pericárdico y vena cava inferior pletórica.

\section{Imagen 1:}

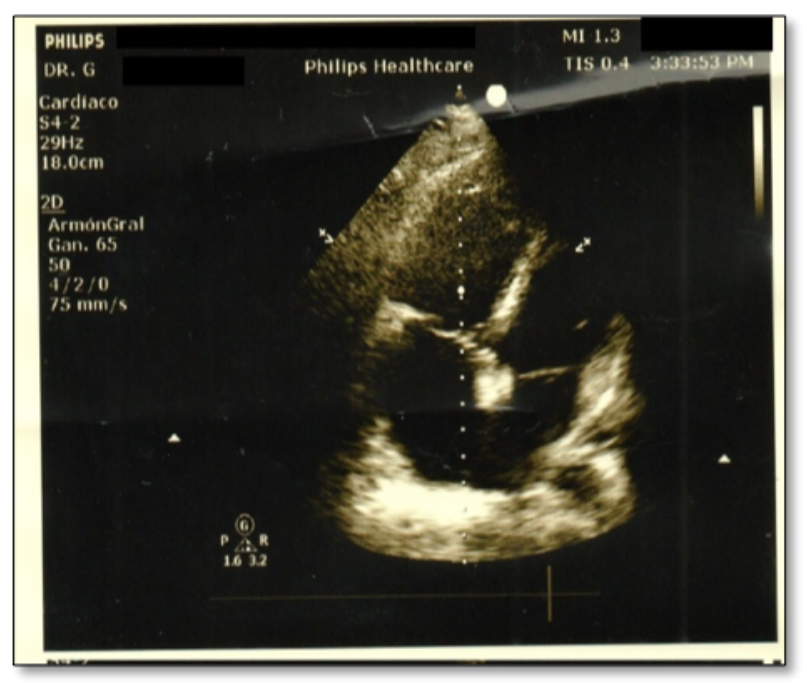


Imagen 2:

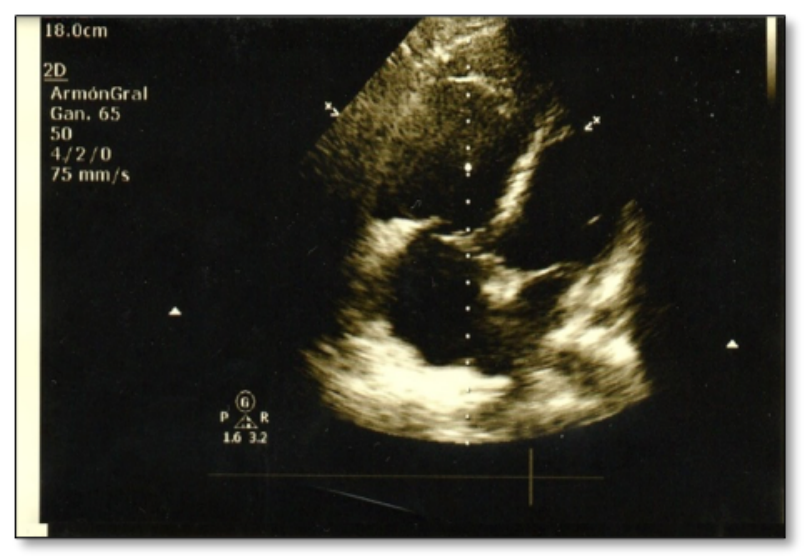

Imagen 3:

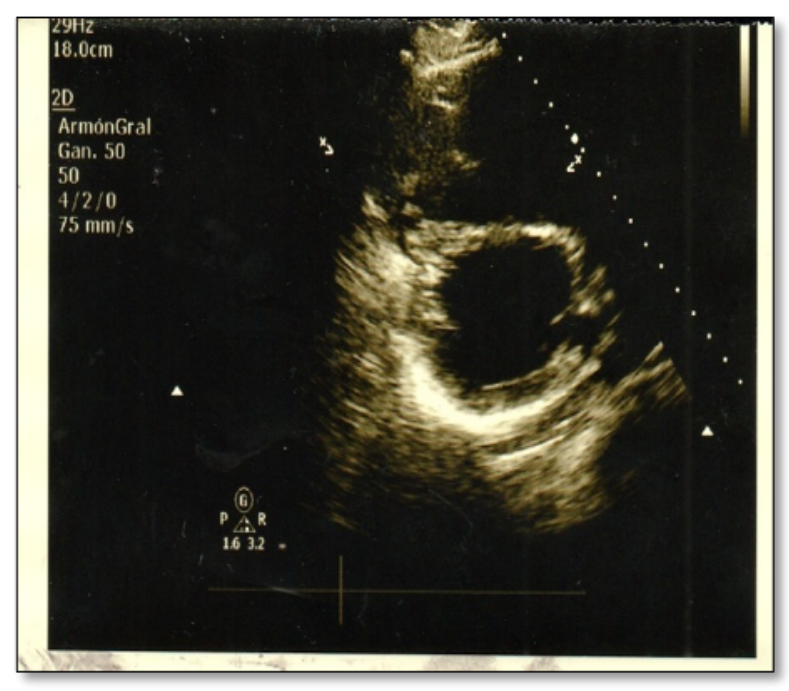

Se solicitó una serie de anticuerpos como parte del estudio de síndrome de hipercoagulabilidad, los cuales reportaron los siguientes resultados: beta - 2 -glicoproteína IgG 619.40; beta - 2 - glicoproteína, IgM menor a 1.1; beta - 2 - glicoproteína, IgA 24.60; complemento sérico fracción C 4 8.11, anticuerpos IgG anticardiolipinas 321.90, anticuerpos IgM anticardiolipinas 2.00. Además, se documenta ANA y anti DNA, Factor Reumatoideo, serologías HIV, VHB, VHC, VDRL y cultivos, negativos.

Se complementan estudios con US Doppler de extremidades inferiores, evidenciando trombosis de vena safena mayor izquierda, con indemnidad de resto de circulación venosa profunda de dichas extremidades así como territorio de cava inferior y sistema porta. Ultrasonido general de abdomen sin alteraciones patológicas así como endoscopía digestiva alta.

Se valora caso por servicio de Reumatología concluyendo que, por los hallazgos clínicos obtenidos, el paciente presenta un Síndrome Antifosfolípido Primario, por lo que se inicia tratamiento con Hidroxicloroqui- 


\section{CIENCIA\&SALUD}

na y traslape a anticoagulación oral con warfarina, una vez mejorada la condición clínica global del paciente. En vista de los hallazgos clínicos en el POCUS compatibles con probable aumento de presión de cavidades derechas, se realiza Ecocardiograma transtorácico reportando: función sistólica de ventrículo derecho disminuida con dilatación severa de cámaras derechas, hipertensión pulmonar severa PSAP 80 mmhg (tipo 4) y una FEVI del 50\%. Por eso, se da inicio al tratamiento con inhibidores de 5-fosfodiesterasa.

\section{Discusión y análisis del caso}

El síndrome antifosfolipídico es una patología autoinmune sistémica que presenta manifestaciones vasculares y obstétricas asociadas a mecánicos inflamatorios y trombóticos mediados por anticuerpos antifosfolípidos.

Los anticuerpos antofosfolípidos son una familia de inmunoglobulinas (IgM, IgG, IgA), las cuales se dirigen contra los fosfolípidos que se encuentran unidos a proteínas plasmáticas, especialmente a la B2 glucoproteína. Estos pueden ser de tres tipos: anticardiolipina, anticoagulante lúpico y beta dos glicoproteína (1).

EI SAF tiene varias clasificaciones, una de estas está basada en las asociaciones del síndrome con otras patologías, en cuyo caso, si hay asociación con una enfermedad subyacente se denomina SAF secundario, si por el contrario el paciente no presenta asociación alguna con otra patología se denomina como SAF primario.

Generalmente, el SAF secundario se relaciona con el lupus eritematoso sistémico y el asociado con enfermedad semejante a LES, donde se engloban las patologías similares a este que no cumplen con los criterios diagnósticos establecidos para definirse como tal.

Otra clasificación es el SAF obstétrico, no obstétrico y basado por título de anticuerpos.

Existen casos donde se documentan autoanticuerpos antifosfolípidos positivos en los que no se desarrollan eventos trombóticos, tales como endocarditis infecciosa, TB, lepra, HIV, sífilis, hepatitis A y Parvovirus (2). En cuanto a la etiología, se han identificado factores genéticos relacionados, en donde el estímulo antigénico desencadena el SAF en una persona genéticamente predispuesta, estos se encuentran principalmente en el complejo mayor de histocompatibilidad.

También se han implicado factores ambientales en el desarrollo del SAF, principalmente factores de origen infecciosos como VIH o sífilis, además se han vinculado varias drogas con el desarrollo de los anticuerpos antifosfolipídicos y eventos trombóticos, como fenotiazida, quinidina, procainamida, interferón B, bloqueadores de canales de calcio y peniclinas sintéticas $(3,4)$.

\section{Epidemiología}

Los anticuerpos fosfolípidos que se unen a proteínas plasmáticas se presentan de 1 a $5 \%$ de la población general $(5,8,9)$. La prevalencia aumenta con la edad. Algunos estudios epidemiológicos han estimado que la incidencia de SAF sería aproximadamente cinco casos nuevos por 10.000 habitantes por año y su prevalencia es de 40 casos por 100.000 habitantes. Existe predominancia de mujeres 5:1 (6).

\section{Fisiopatología}

Numerosos estudios apoyan la importancia de la activación de los monocitos, células endoteliales, plaquetas y complemento, así como la inducción de un estado protrombótico causado por la interferencia con las 


\section{CIENCIA\&SALUD}

proteínas de la cascada de la coagulación (5).

Los anticuerpos antifosfolipídicos consisten en un grupo heterogéneo de autoanticuerpos, los cuales interactúan con gran cantidad de proteínas plasmáticas de unión con fosfolipídicos, principalmente b2- glicoproteina 1, protrombina, trombomodulina, cininógenos, antitrombina III, proteína C, proteína S, anexina I,II y $\vee$.

El fosfolípido más importante para el cual estas proteínas se unen es la fosfatidilserina, ubicado en la superficie interna de la membrana celular. Se exterioriza en membranas celulares activadas o apoptóticas, incluidos los trofoblastos, monocitos endoteliales, lo que resulta en la activación celular, aclaramiento de células apoptóticas y coagulación $(10,11)$.

Manifestaciones clínicas

Las manifestaciones clínicas mayoritariamente son trombosis venosas (TVP en un 39\%, livedo reticular $24 \%$ y embolia pulmonar en $14 \%$ ) y arteriales (apoplejía en un 20\%, engrosamiento y disfunción de válvulas cardíacas, vegetaciones de Libman -Sacks (12) o ambos trastornos 14\%, episodio isquémico transitorio 11\%). Se presentan manifestaciones neurológicas, de origen incierto como la migraña en 20 \%, seguido por epilepsia en $7 \%$; renales en 3\%, osteoarticulares, como artralgias en 39\% y artritis en $27 \%$. También hay manifestaciones obstétricas como preeclampsia en $10 \%$ y eclampsia en $4 \%$ y pérdidas fetales precoces en $35 \%$ (13, 14).

\section{Diagnóstico}

El reconocimiento temprano de Síndrome Antifosfolipídico (SAF) es crucial, ya que el tratamiento puede reducir la mortalidad y la morbilidad, sin embargo, el SAF no se diagnostica lo suficiente, a menudo, debido a su clínica tan variable combinada con una falta de estandarización de pruebas de diagnóstico (3).

Se debe considerar posibilidad de SAF en casos de pacientes que presenten trombosis, apoplejía en personas menores de 55 años de edad o en mujeres con complicaciones en el embarazo, abortos a repetición, livedo reticular como manifestación cutánea o trombocitopenia. En estos casos se hace necesario medir los anticuerpos antifosfolípidos mediante prueba de laboratorio. La presencia de un criterio clínico, por lo menos, y uno de los criterios de laboratorio, sirve como diagnóstico, aunque haya otras causas de trombofilia.

\section{Los criterios clínicos son (4):}

1. Trombosis vascular (uno más episodios de trombosis arterial o venosa en cualquier órgano o tejido).

\section{Complicaciones en el embarazo que incluyen:}

a) Una muerte o más, no explicadas, de un feto morfológicamente normal o posterior a la décima semana de gestación.

b) Uno o más nacimientos prematuros de un recién nacido morfológicamente normal antes de las 34 semanas de gestación producto de preeclampsia, eclampsia o insuficiencia placentaria.

c) Tres o más abortos espontáneos consecutivos, sin causa definida antes de la décima semana de gestación.

\section{Criterios de laboratorio:}

a) Anticoagulantúe lúpico, b) anticardiolipina, c) anticuerpo contra B2GPI o los últimos dos elementos men- 


\section{CIENCIA\&SALUD}

cionados, con valores intermedios o altos en dos ocasiones, con una diferencia de 12 semanas.

El riesgo de complicaciones relacionadas con SAF aumenta de acuerdo con el nivel de titulación de anticuerpos involucrados, implicando así un mayor riesgo de tromboembolismo y morbilidad en el embarazo.

\section{TRATAMIENTO}

La base del tratamiento para pacientes con SAF es la prevención de trombosis (tromboprofilaxis). La positividad de anticuerpos antifosfolípido puede ocurrir en dos situaciones clínicas principales: presencia de anticuerpos antifosfolípido en sujetos sin trombosis previa (tromboprofilaxis primaria), o pacientes con SAF que ya han tenido un evento trombótico (tromboprofilaxis secundaria) (3, 7, 11).

La tromboprofilaxis primaria se centra principalmente en el estricto control de cualquier factor de riesgo vascular adicional (tal como hipercolesterolemia, obesidad, sedentarismo, tabaquismo). Se recomiendan anticonceptivos, que contengan solo progestágeno por considerarse de mayor seguridad y evitar anticonceptivos orales que contienen estrógenos. Asimismo, se recomienda tromboprofilaxis, durante situaciones de alto riesgo (cirugía, posparto, inmovilización prolongada). $(16,17,18)$

Posterior a la primera crisis, se administra warfarina de modo permanente a los pacientes con SAF, pues se intenta alcanzar un INR entre 2.5 y 3.5, sola o en combinación con 80 mg de AAS por día.

Las complicaciones del embarazo se evitan por medio de una combinación de heparina 80mg de AAS cada $24 \mathrm{hrs}$; la inmunoglobulina intravenosa, en razón de $400 \mathrm{mg} / \mathrm{kg}$ de peso por día por 5 días se utiliza para prevención de abortos $(19,20)$.

Los pacientes que presentan anticuerpos antifosfolipídicos, sin complicaciones clínicas, positivos de formar simultánea para anticuerpo anticardiolipina, anti B2GPI y anticoagulante lúpico o con LES tiene riesgo de padecer episodios trombóticos y se tratan con 80 mg de AAS al día.

Pacientes con SAF presentan crisis recidivantes a pesar del tratamiento anticoagulante adecuado. Puede ser beneficiosos administrar inmunoglobulina intravenosa $400 \mathrm{mg} / \mathrm{kg}$ de peso por día por cinco días, en estos casos. El paciente con SAFC, en Unidad de Cuidados Intensivos, utiliza HBPM en dosis terapéuticas. Los inhibidores de factor $X$ activado, ligado con fosfolípido, como fondaparinux, en razón de $7.5 \mathrm{mg}$ por vía SC, todos los días, o rivaroxaban, en dosis de $10 \mathrm{mg}$ por día VO, son eficaces, en el caso de pacientes con trombocitopenia y síndrome de trombosis inducida por heparina (9).

\section{CONCLUSIONES}

El tromboembolismo pulmonar es una de las manifestaciones más letales del síndrome antifosfolipídico, dentro de la gran diversidad de variabilidad de presentaciones de la enfermedad.

Con respecto al presente caso, la sospecha diagnóstica radica en la presentación de TEP Masivo en un paciente masculino joven, sin evidencia de enfermedades infecciosas, neoplásicas ni reumatológicas concomitantes, por lo que el trabajo diagnóstico se encaminó a la comprobación de pruebas que pudieran identificar de primera mano, la posibilidad que el paciente tuviera Síndrome Antifosfolípido Primario, siendo así un diagnóstico poco común en nuestro medio, por la incidencia mucho mayor de la enfermedad en el sexo femenino. El tratamiento se basa en evitar la recurrencia de fenómenos trombóticos predominantemente, esto con anticoagulación oral y además, en la rehabilitación cardiopulmonar por la hipertensión pulmonar residual secundaria al TEP así como el uso concomitante de inhibidores de la 5-fosfodiesterasa 


\section{REFERENCIAS BIBLIOGRÁFICAS}

1. Manzano AC. García OM. Valor pronóstico de la escala PESI y Angiotac en pacientes con TEP en el Hospital Universitario San Ignacio. Rev. Colomb. Radiol. 2016; 27(3): 4481-5

2. Barva JR. Síndrome de anticuerpos antifosfolípido. Rev. Mex. Pat. Clin. 2003; 50(1):20-32.

3. Cruz DG. síndrome de anticuerpos antifosfolipidicos;patogénesis, diagnóstico y tratamiento. Rev Hematol Mex. 2016 octubre;17(4):256-261.

4. Rojas SJ. Revisión síndrome de anticuerpos antifosfolípido. Rev. Mex. Reumat 1996; 11: 179-185.

5. Wilson WA et al. Genetic risk factors for aPL syndrome. Lupus. 1996; 5: 398-403.

6. Triplett DA. Lupus anticoagulants/antiphospholipid-protein antibodies: the great imposters. Lupus 1996; 5: 431-435.

7. Negrini S, Pappalardo F, Murdaca G, Indiveri F, Puppo F. The antiphospholipid syndrome: from pathophysiology to treatment. Clin Exp Med. 2017;17(3):257-267.

8. Kasper D Braunwald E Fauci A Hauser S Longo D Jameson J. Harrison's Principles of Internal Medicine. Mc Graw Hill Companies.Inc.New Cork-U.S.A. 20th edición. 2019. (4) 2526-2527.

9. Hwang J Shin S Kim Y et al. Epidemiology of Anthiphospholipid Syndrome in Korea: a Nationwide Population-based Study. JKMS Sci.2020 Feb 10; 35(5): 1-12

10. Alfaro R.Sindrome antifosfolipídico(Revisión bibliográfica).Revista Médica Costa Rica y Centroamérica LXVI(589) 313-317 2009

11. Rodriguez M Montero C Verea H. Hemorragia pulmonar y síndrome antifosfolipídico primario: aportación de un caso y revision de la literatura.An Med Interna ( Madrid) Vol 24 Numero 3 pp 125128,2007 .

12. J. Ma. Calvo J. C. Bureo Dacal, J. L. Ramos, P. Bureo Dacal, M. Pérez Miranda. Síndrome antifosfolipídicoprimario: características y evolución de una serie de 17 AN. MED. INTERNA (Madrid) Vol. 19, N.o 5, pp: 226-229, 2002.

13. Tai-Chin Hsieh. Po-Ren Hsueh. Fang-Lan Yu. Shio-Shin Jean. Fu-Lun Chen. Tsong-Yih Ou. WenSen Lee. Co-occurrence of Leriche syndrome and antiphospholipid syndrome in a man with refractory ulcers of the lower limbs. Journal of Microbiology, Immunology and Infection.(2016) 49, páginas: 309 a 310.

14. Cervera R, Piette JC, Font J, et al. Antiphospholipid syndrome: clinical and immunologic manifestations and patterns of disease expression in a cohort of 1,000 patients. Arthritis Rheum. 2002;46(4):1019-27. 


\section{CIENCIA\&SALUD}

15. Ruiz-Irastorza G, Crowther M, Branch W, Khamashta MA. Antiphospholipid syndrome. Lancet. 2010;376(9751):1498-509.

16. Cohen D, Berger SP, Steup-Beekman GM, Bloemenkamp KW, Bajema IM. Diagnosis and management of the antiphospholipid syndrome. BMJ. 2010;340:c2541.

17. Sciascia S, Cuadrado MJ, Khamashta M, Roccatello D. Renal involvement in antiphospholipid syndrome. Nat Rev Nephrol. 2014;10(5):279-8.

18. Vargas F Pinto LF Donado $\mathrm{J}$ et al.Síndrome Antifosfolipidico:morbilidad y evolución de una cohorte de pacientes del Hospital Pablo Tobón Uribe de Medellín-Colombia.Revista Colombiana de Reumatología Vol.13 No.2 Junio 2006,pp.109-119.

19. Maggiorini M, Knoblauch A, Schneider J, Russi EW. Diffuse microvascular pulmonary thrombosis associated with primary antiphospholipid antibody syndrome Eur Respir J 1997; 10: 727-30.

Greaves M, Cohen H, Machine SJ, Mackie I. Guidelines on the investigation and management of the antiphospholipid syndrome. Br J Haematol 2000; 109: 704-15 